\title{
VOLTAGE DEPENDENCE OF SUPERCAPACITOR CAPACITANCE
}

\author{
Arkadiusz Szewczyk ${ }^{1)}$, Josef Sikula ${ }^{2)}$, Vlasta Sedlakova ${ }^{2)}$, Jiri Majzner ${ }^{2)}$, Petr Sedlak²), \\ Tomas Kuparowitz ${ }^{2}$
}

1) Gdańsk University of Technology, Faculty of Electronics, Telecommunications and Informatics, Narutowicza 11/12, 80-233 Gdańsk, Poland》szewczyk@eti.pg.gda.pl,+4858347 2140)

2) Brno University of Technology, Central European Institute of Technology, Technicka 10, Brno 616 00, Czech Republic (sikula@feec.vutbr.cz,sedlaka@feec.vutbr.cz,majzner@feec.vutbr.cz,sedlakp@feec.vutbr.cz,xkupar01@stud.feec.vutbr.cz)

\begin{abstract}
Electronic Double-Layer Capacitors (EDLC), called Supercapacitors (SC), are electronic devices that are capable to store a relatively high amount of energy in a small volume comparing to other types of capacitors. They are composed of an activated carbon layer and electrolyte solution. The charge is stored on electrodes, forming the Helmholtz layer, and in electrolyte. The capacitance of supercapacitor is voltage- dependent. We propose an experimental method, based on monitoring of charging and discharging a supercapacitor, which enables to evaluate the charge in an SC structure as well as the Capacitance-Voltage $(\mathrm{C}-\mathrm{V})$ dependence. The measurement setup, method and experimental results of charging/discharging commercially available supercapacitors in various voltage and current conditions are presented. The total charge stored in an SC structure is proportional to the square of voltage at SC electrodes while the charge on electrodes increases linearly with the voltage on SC electrodes. The Helmholtz capacitance increases linearly with the voltage bias while a sublinear increase of total capacitance was found. The voltage on SC increases after the discharge of electrodes due to diffusion of charges from the electrolyte to the electrodes. We have found that the recovery voltage value is linearly proportional to the initial bias voltage value.
\end{abstract}

Keywords: Electronic Double-Layer Capacitor (EDLC), charge diffusion, recovery voltage.

C 2016 Polish Academy of Sciences. All rights reserved

\section{Introduction}

Although the double-layer capacitance has been known since the middle of XIX century, its electrical behaviour is still not fully understood. A completely discharged supercapacitor can be charged to the nominal voltage in a few seconds, but then the voltage at the SC terminals drops due to redistribution of the charge by diffusion in the SC structure. Whereas the time constant of charging the electrodes is of the order of seconds, the time constant of diffusion processes is of the order of a hundred seconds. Apparently, such a behaviour differs from those that occur in conventional types of capacitors. Thus, the traditional models used to describe the capacitor behaviour are inadequate to the electrochemical capacitors [1,2]. A number of equivalent circuit models of various complexity have been proposed to predict or describe $\mathrm{SC}$ behaviour models under numerous conditions in power storage applications required by designers and engineers, e.g. [3-7]. However, only a few of them took into account the charge redistribution phenomenon. A simplified electric model of supercapacitor consisting of two branches of RC elements including one resistor with a time-dependent resistance is proposed in [1] to forecast the charge redistribution process in an SC structure for days (see Fig. 1). The first branch (capacitor $C_{1}$ and resistor $R_{1}$ ) represents the Helmholtz capacitance, $C_{H}$, with an Equivalent Series Resistor (ESR), the second one (capacitor $C_{2}$ and resistor $R_{2}(t)$ ) represents the diffuse capacitance, $C_{\mathrm{D}}$, and the resistance for charge transport by diffusion, $R_{\mathrm{d}} . R_{\mathrm{L}}$ represents the leakage resistance. 


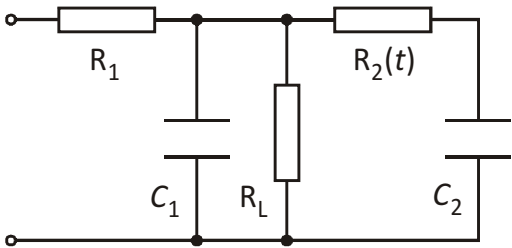

Fig. 1. A simplified electric model of supercapacitor [1]

The value of resistance $R_{\mathrm{d}}$ increases with the square root of time. During charging the supercapacitor up to its nominal voltage, as the value of ESR is very low, the $C_{\mathrm{H}}$ capacitor can be charged within few seconds. Then the charge is redistributed by diffusion in the supercapacitor structure, which can be described by charging the capacitance $C_{\mathrm{D}}$ through the time-dependent diffuse resistance $R_{\mathrm{d}}$ with the time constant of the order of a hundred seconds. The charge transferred to the SC during the charging is higher than the charge which can be delivered from the electrodes during the SC short-circuiting. It is known that the complete charging of SC requires a period of a few hundred hours [6-8] due to the diffusion processes. Also, the discharging SC lasts several days due to a certain amount of charge always remaining stored inside the SC, even after a long-term SC discharging [8]. The charge redistribution of a so-called "residual charge" plays the key role in a general SC behaviour [6]. This charge redistribution is due to a limited ionic mobility and hence a high resistance of ions moving in the vicinity of carbon electrodes [7]. It is very well known that the nonlinearity of voltage-time dependence during the charging SC by the constant current is caused by the dependence of SC capacity on the electric field $[4,9,10]$. Concerning this fact, the charge storing and charge discharging are required to be precisely measured to evaluate the $\mathrm{SC}$ and its behaviour. In this work we present an experimental setup and a novel technique which enables to estimate the total charge delivered to the SC during the long-term charging and quick discharging. This quick discharging simulates the SC short-circuiting if only the charge from electrodes is discharged. From the bias voltage and estimated total charge for SC charging the total capacitance of SC is calculated, while from the charge delivered from SC during the quick discharge the capacitance of electrodes - the Helmholtz capacitance - is calculated. The dependences of charge and capacitance on the bias voltage are also presented.

\section{Experiment}

\subsection{Measured samples}

The experiments were performed on two different types of commercially available supercapacitors:

- NessCap Part Number ESHSR-0010C0-002R7, capacitance 10 F, rated voltage 2.7 V. Two sets of samples denoted N100 (produced in 2013) and N200 (produced in 2014) were studied. Each set contained 10 samples.

- CapXX Product Name HS 130F, capacitance 2.4 F, rated voltage 2.75 V. A set of samples denoted XX100 (produced in 2013) was studied. The set contained 6 samples.

\subsection{Measurement setup}

The measurement setup for charging/discharging supercapacitors is shown in Fig. 2a. The system is composed of a measurement unit (Fig. 2b), a current/voltage source and a computer for the measurement control and signal acquisition. 
The measurement unit is equipped with two electro-magnetic keys, $S 1$ and $S 2$, for switching the supercapacitor between the energy source for charging and the ground - for discharging. As the keys two Zettler AZ943 relays with a contact resistance below $100 \mathrm{~m} \Omega$ were used. The Device Under Test (DUT) is charged through a low resistance $R 1$ for current measurement. In the experiment, a wire-wound $2,2 \Omega$ resistor with a power dissipation $P_{\mathrm{d}}=20 \mathrm{~W}$, was used. The charging current $I_{\mathrm{C}}(t)$ varies in time and its value is:

$$
I_{\mathrm{C}}(t)=[V 1(t)-V 2(t)] / R 1 .
$$

$V 1(\mathrm{t})$ is the voltage measured at the serial connection of resistor $R 1$ and DUT, whereas $V 2(t)$ is the voltage measured directly at the SC device terminal, while the second terminal is connected to the ground. The total charge $Q_{\mathrm{T}}$ delivered to the $\mathrm{SC}$ during the charging is calculated by integration of the charging current over time.

a)

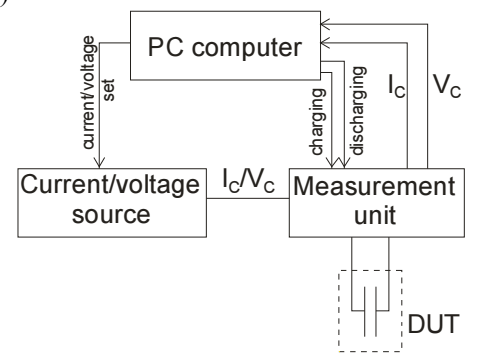

b)

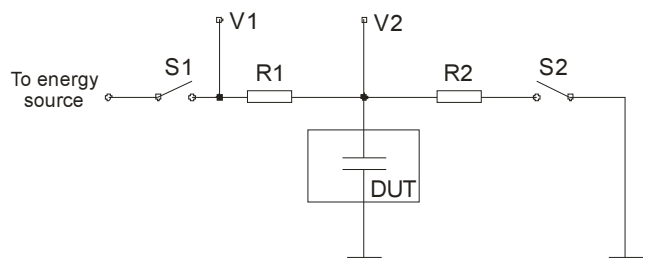

Fig. 2. a) The measurement setup for charging/discharging supercapacitors;

b) the detailed scheme of measurement unit.

The DUT can be discharged either by short-circuiting with a small resistance $R 2$ using $S 2$ key or with a negative current pulse generated by the current source. The discharging lasts until the voltage on the terminals drops to zero (for safety reasons, the discharging is finished when the voltage at the SC terminals drops below 20 millivolts). In our case the device is discharged through a wire-wound $0,22 \Omega$ resistor, which can be easily replaced in order to establish proper discharge conditions. The time-dependent discharging current $I_{\mathrm{D}}(t)$ value is:

$$
I_{\mathrm{D}}(t)=V 2(t) / R 2 .
$$

The DUT discharge time cannot exceed approximately 20 seconds in order to discharge the charges from electrodes only. For a longer discharge time also the charges transferred in the structure by means of the diffusion mechanism are discharged. The discharge resistor can be replaced by an active load.

The charge $Q_{\text {Dis }}$ transferred from the $\mathrm{SC}$ during the discharging is calculated by numerical integration of $I_{\mathrm{D}}(t)$ over time.

As an energy source either the voltage or the current source can be used. In our case the device is charged by a programmable voltage source with a current limit to protect SC against damage. The charging/discharging procedure is controlled by a computer program, which controls the state of relays and monitors the charging current and SC voltage. The voltages $V 1$ and $V 2$ are measured by a National Instruments USB-6612 data acquisition card with a 16-bit $\mathrm{AD}$ converter with $0,153 \mathrm{mV}$ resolution and $50 \mathrm{~ms}$ sampling period. The charging current and SC voltage values obtained at different moments are stored in a text file for further analysis. 


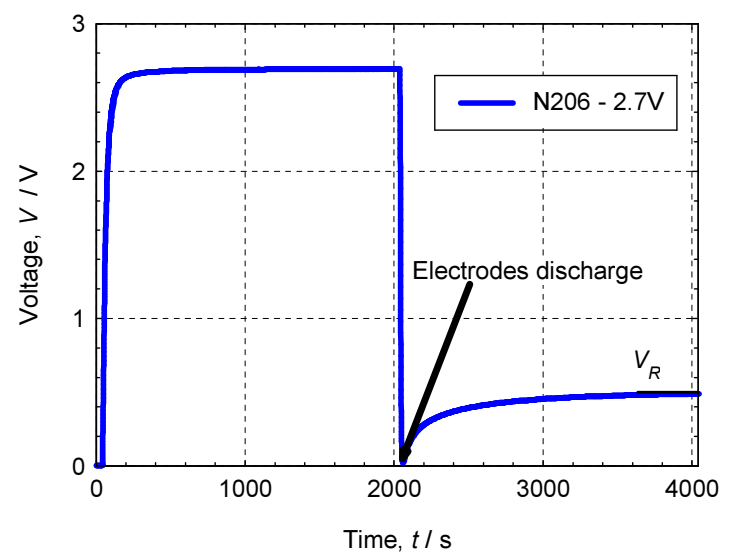

Fig. 3. A typical shape for the charging/discharging procedure; $V_{\mathrm{R}}$ represents the recovery voltage value measured at $2000 \mathrm{~s}$.

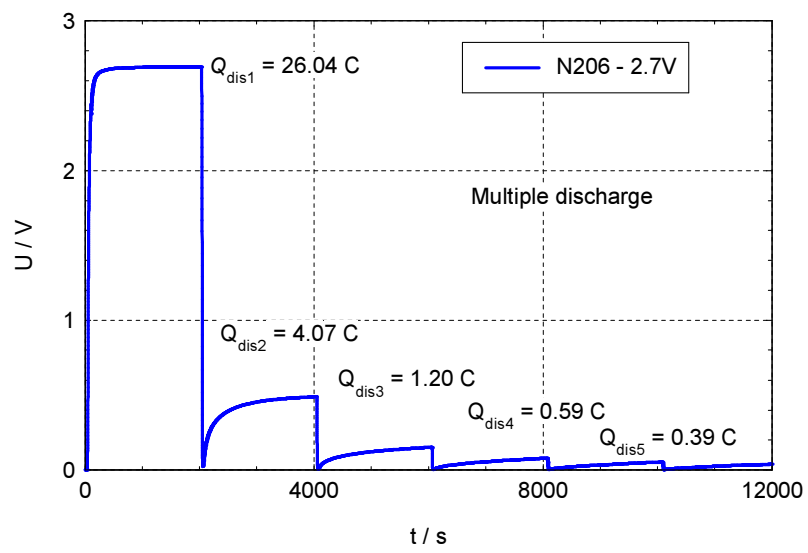

Fig. 4. An experiment of the multiple discharge of electrodes, measured for an N206 sample from the N200 series.

A typical shape for the charging/discharging procedure is shown in Fig. 3. The DUT had been charged to the appropriate bias voltage value for 2000 seconds. During this time the charging current $I_{\mathrm{C}}(t)$ was monitored and then the total charge $Q_{\mathrm{T}}$ was calculated. After $2000 \mathrm{~s}$ the DUT was discharged within a time interval of $20 \mathrm{~s}$, and the discharge current $I_{\mathrm{D}}(t)$ and charge $Q_{\text {Dis }}$ values were determined. After discharging the electrodes the voltage at DUT terminals had been monitored for additional 2000 seconds. At the end of this time interval the recovery voltage $V_{\mathrm{R}}$ value was determined. The voltage at the SC terminal increases due to redistribution of the residual charge - given as $Q_{\mathrm{R}}=Q_{\mathrm{T}}-Q_{\text {Dis }}-$ in the structure by diffusion, leading to the appearance of free charges on the SC terminals.

In order to determine the dependence of charge $Q_{\text {Dis }}$ on the voltage at SC terminals we made an experiment with a multiple discharge of the supercapacitor (Fig. 4). After the initial charging/discharging procedure and monitoring the recovery voltage for $2000 \mathrm{~s}$, the sample had been discharged (for $20 \mathrm{~s}$ ) and the voltage had been monitored for additional $2000 \mathrm{~s}$. This additional discharging and voltage monitoring was repeated additional 4 times. The total charge transferred to the $\mathrm{N} 206$ supercapacitor during the initial charging was $Q_{\mathrm{T}}=36.72 \mathrm{C}$; the charges $Q_{\text {Dis }}$ within each discharge are shown in Fig. 4 . After calculation - by subtracting those charge 
values from the initial charge $Q_{\mathrm{T}}-$ we have found that after the 5 th discharge there still remains a residual charge $Q_{\mathrm{R}}=4.43 \mathrm{C}$ in the structure.

\subsection{Capacitance estimation}

The total capacitance $C_{\mathrm{T}}$ of the supercapacitor can be calculated from the total charge, $Q_{\mathrm{T}}$, delivered to the SC during 2000 seconds of charging to a given voltage $V$ :

$$
C_{\mathrm{T}}=Q_{\mathrm{T}} / V \text {. }
$$

The capacitance of SC electrodes, corresponding to the capacitance $C_{\mathrm{H}}$ of Helmholtz layer formed on the electrode - electrolyte interface, can be calculated from the discharge charge $Q_{\text {Dis }}$ transferred from the electrode within the controlled short-circuiting of SC with the voltage $V$ at its terminals:

$$
C_{\mathrm{H}}=Q_{\mathrm{Dis}} / V \text {. }
$$

\section{Results and discussion}

The dependence of the total charge transferred to the SC during a time interval of 2000 seconds (of charging on a given bias voltage value) on the bias voltage is shown in Fig. 5 for samples of N100, N200, and XX100 sets, respectively. The measured data are approximated by the equation:

$$
Q=a V^{2}+b V
$$

where $a$ and $b$ are constants. Their values are given in Fig. 5 for all fitted dependences. We can see that the fitted dependence matches precisely the experimental data for all examined sets of samples. The total charge is proportional to the sum of linear and quadratic components of voltage at the electrodes.

Figures 6 and 7 show the dependence of the charge $Q_{\text {Dis }}$ discharged from the SC electrodes within the time intervals: $t_{\text {dis }}=20 \mathrm{~s}$ for NessCap samples (N100 and N200 series, and data from the multiple discharge of N105, N201 and N206 samples), and $t_{\text {dis }}=10 \mathrm{~s}$ for CapXX samples (XX100 series), respectively, on the voltage at the SC electrodes in the linear (Fig. 6) and logarithmic (Fig. 7) scale. The slope $m$ of linear approximation of measured data in the log-log scale in Fig. 7 equals to a value of 1.06 that indicates a linear dependence of the charge $Q_{\text {Dis }}$ on the voltage at the SC electrodes. The slope $m$ value of linear approximation of measured data in the linear scale (Fig. 6) corresponds to the nominal capacitance of evaluated samples.

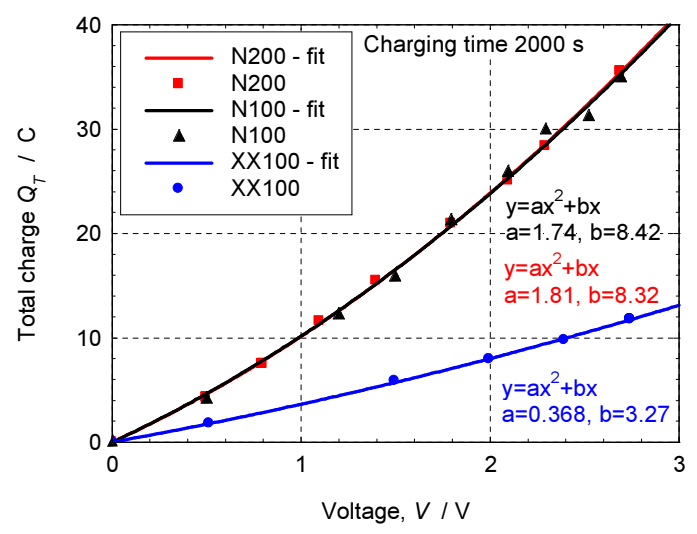

Fig. 5. The total charge transferred to the SC (during 2000 seconds on a given bias voltage value) vs. the voltage bias for samples of N100, N200, and XX100 sets, respectively. 


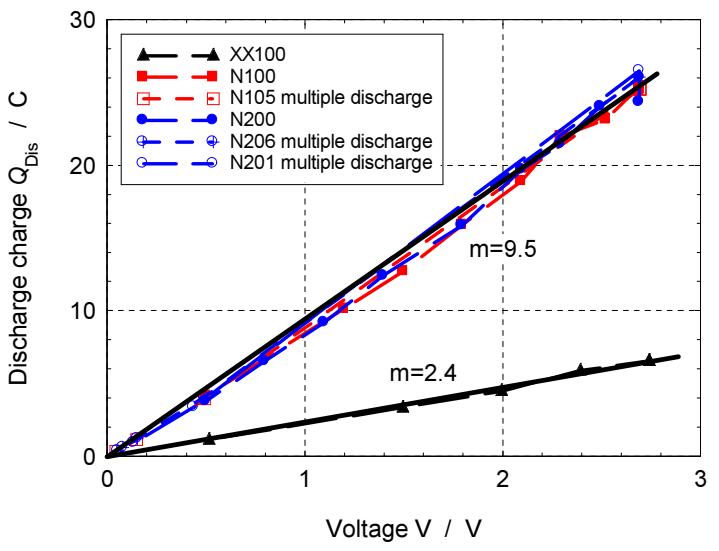

Fig. 6. The charge discharged from the SC electrodes vs. the voltage at SC electrodes for N100 and N200 series (the discharge time $t_{\mathrm{dis}}=20 \mathrm{~s}$ ), and XX100 series (the discharge time $t_{\mathrm{dis}}=10 \mathrm{~s}$ ), respectively.

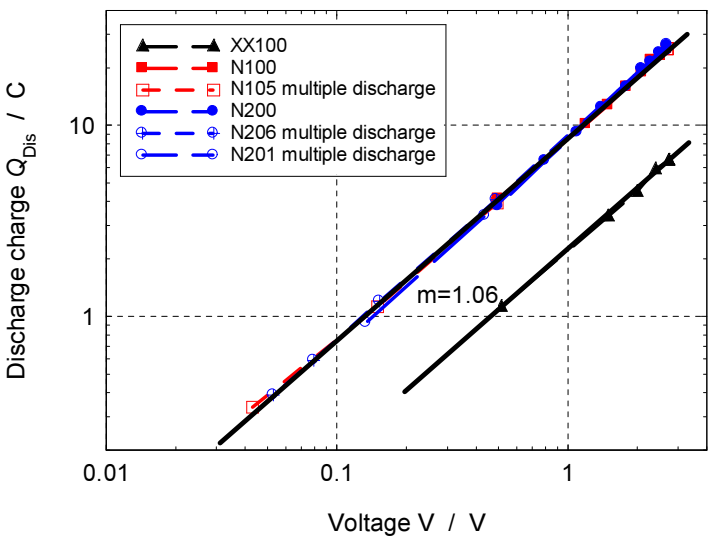

Fig. 7. The charge discharged from the SC electrodes vs. the voltage at SC electrodes in the log-log scale for samples of N100, N200, and XX100 sets, respectively. The value of slope $m=1.06$ shows a linear dependence of $Q_{\text {Dis }}$ on $V$.

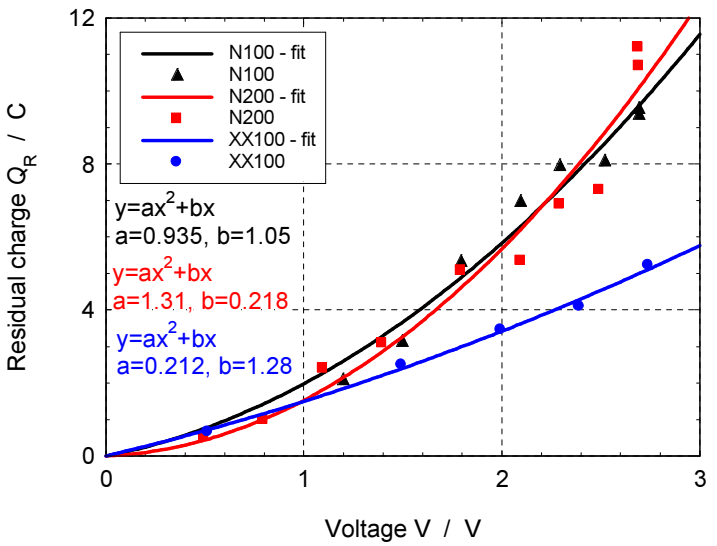

Fig. 8. The residual charge remaining in the SC structure after the discharge of electrodes vs. the bias voltage for samples of N100, N200, and XX100 sets, respectively. 
The dependence of the residual charge, remaining in the SC structure after the discharge of electrodes, on the bias voltage is shown in Fig. 8. The residual charge corresponds to the charge stored in the diffuse capacitance $C_{\mathrm{D}}$ during the initial charging [1]. The measured data can be approximated by (5). The residual charge in the SC structure is proportional to the sum of linear and quadratic components of voltage at the electrodes.

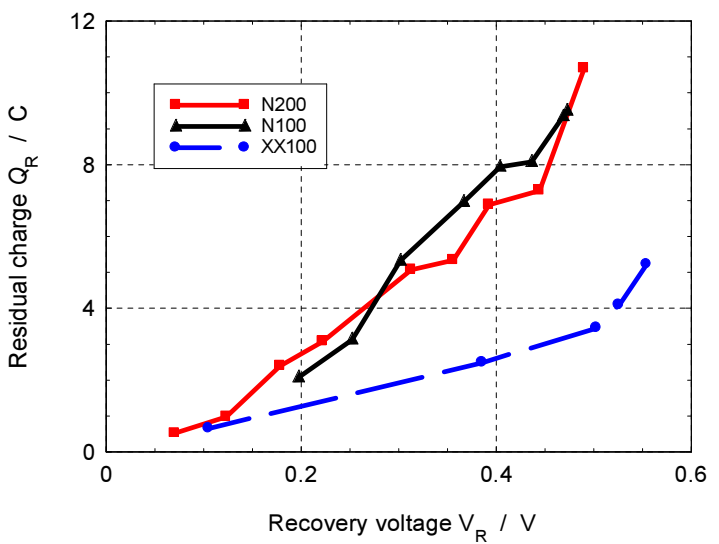

Fig. 9. The residual charge remaining in the SC structure after the discharge of electrodes vs. the recovery voltage $V_{\mathrm{R}}$ measured $2000 \mathrm{~s}$ after the discharge of electrodes for samples of N100, N200, and XX100 sets, respectively.

Figure 9 shows the dependence of the residual charge, remaining in the SC structure after the discharge of electrodes, on the recovery voltage $V_{\mathrm{R}}$ measured $2000 \mathrm{~s}$ after the discharge of electrodes for samples of N100, N200, and XX100 sets, respectively. This dependence is super-linear and this fact indicates that the capacitance associated with diffusion of charges in the SC structure increases with the electric field.

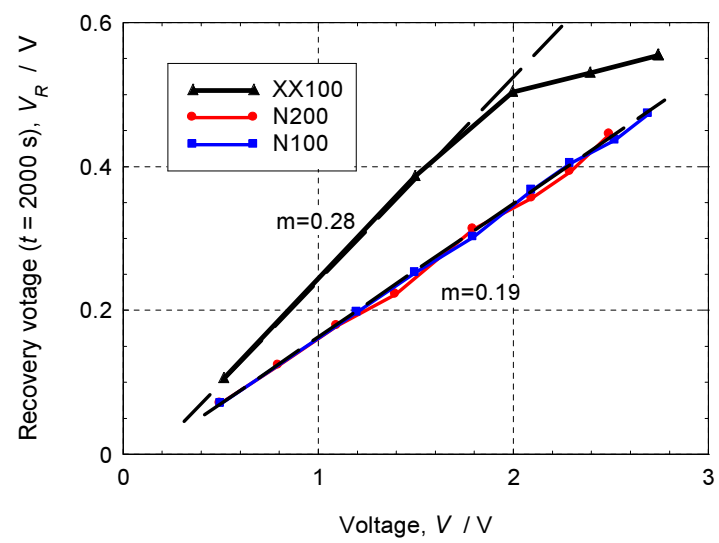

Fig. 10. The recovery voltage $V_{\mathrm{R}}$ measured $2000 \mathrm{~s}$ after the discharge of electrodes vs. the bias voltage for samples of N100, N200, and XX100 sets, respectively.

The recovery voltage $V_{\mathrm{R}}$, measured $2000 \mathrm{~s}$ after the discharge of electrodes, exhibits a sublinear dependence on the bias voltage for samples of XX100 set, as shown in Fig. 10. This dependence increases linearly in the whole voltage range for samples of N100 and N200 sets, respectively. It seems that the recovery voltage value could be limited by a certain technological parameter of CS. 


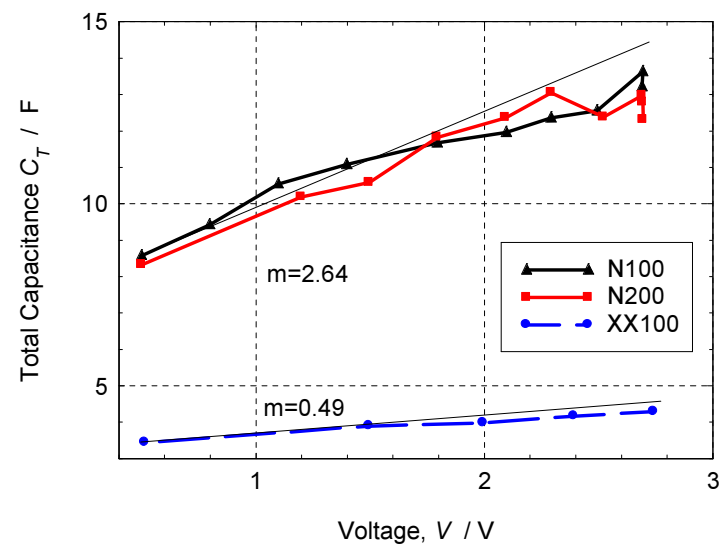

Fig. 11. The total capacitance vs. the bias voltage for samples of N100, N200, and XX100 sets, respectively.

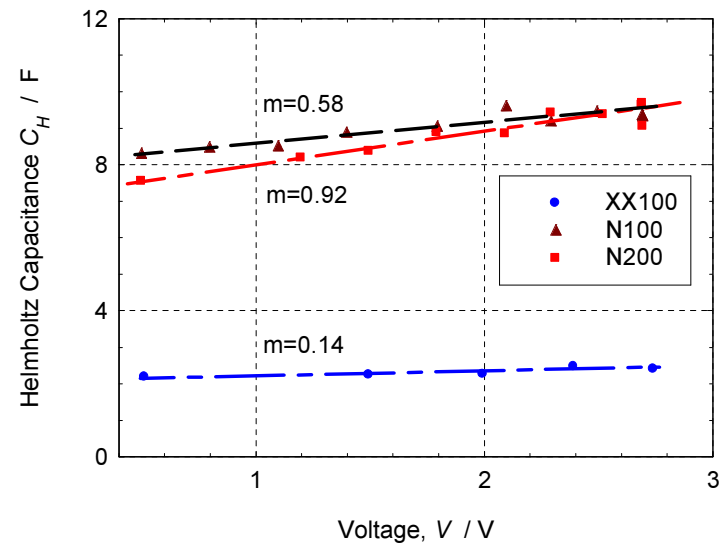

Fig. 12. The Helmholtz capacitance vs. the bias voltage for samples of N100, N200, and XX100 sets, respectively.

The calculated values of total capacitance $C_{\mathrm{T}}$ and Helmholtz capacitance $C_{\mathrm{H}}$ vs. the bias voltage are shown in Figs. 11 and 12, respectively, for samples of N100, N200, and XX100 sets. We can see that the total capacitance increases sub-linearly with the bias voltage for all evaluated series of samples, while the Helmholtz capacitance is linearly proportional to the bias voltage for all examined series.

\section{Conclusion}

A measurement setup and a method for precise evaluation of the charge/discharge processes in the supercapacitor structures were designed. By monitoring the charging current within a time interval of 2000 seconds we can evaluate the total charge stored in the structure and its total capacitance, respectively. The experimental data for the dependence of $Q_{\mathrm{T}}$ on the bias voltage precisely match the sum of linear and quadratic components of voltage at the electrodes. The dependence of the total capacitance on the bias voltage shows a slightly sub-linear behavior.

A quick discharge simulates the SC short-circuiting if only the charge from electrodes is discharged. The discharged charge $Q_{\text {Dis }}$ linearly increases with the bias voltage and the slope value of linear approximation corresponds to the nominal capacitance of evaluated samples. 
The calculated values of Helmholtz capacitance increase linearly with the bias voltage for all examined series of samples.

Besides, we determined the dependence of the residual charge, remaining in the SC structure after the discharge of electrodes, on the bias voltage value. The residual charge in the SC structure corresponds to the charge redistributed by diffusion during the initial charging process and it is proportional to the sum of linear and quadratic components of voltage at the SC terminals.

The recovery voltage $V_{\mathrm{R}}$ (measured $2000 \mathrm{~s}$ after the discharge of electrodes) increases linearly with the voltage bias for NessCap samples, whereas saturation of the $V_{\mathrm{R}}$ value is observed for CapXX samples. It seems that the recovery voltage value could be limited by a certain technological parameter of CS.

\section{Acknowledgement}

Research described in this paper was financed by the National Sustainability Program under grant LO1401. In the research, the SIX Center infrastructure was used.

\section{References}

[1] Sedlakova, V., Sikula, J., Majzner, J., Sedlak, P., Kuparowitz, T., Buergler, B., Vasina, P. (2015). Supercapacitor equivalent electrical circuit model based on charges redistribution by diffusion. J. Power Sources, 286, 58-65.

[2] Sharma, P., Bhatti, T.S. (2010). A review on electrochemical double-layer capacitors. Energy Convers. Manag., 51, 2901-12.

[3] Zubieta, L., Bonert, R. (2000). Characterization of double-layer capacitors for power electronics applications. IEEE Trans. Ind. Appl., 36, 199-205.

[4] Belhachemi, F., Rael, S., Davat, B. (2000). A physical based model of power electric double-layer supercapacitors. Conference Record of the 2000 IEEE Industry Applications Conference, 5, 3069-76.

[5] Faranda, R. (2010). A new parameters identification procedure for simplified double layer capacitor twobranch model. Electr. Power Syst. Res., 80, 363-71.

[6] Torregrossa, D., Bahramipanah, M., Namor, E., Cherkaoui, R., Paolone, M. (2014). Improvement of Dynamic Modeling of Supercapacitor by Residual Charge Effect Estimation. IEEE Trans. Ind. Electron., $61,1345-54$.

[7] Graydon, J.W., Panjehshahi, M., Kirk, D.W. (2014). Charge redistribution and ionic mobility in the micropores of supercapacitors. J. Power Sources, 245, 822-9.

[8] Kaus, M., Kowal, J., Sauer, D.U. (2010). Modelling the effects of charge redistribution during self-discharge of supercapacitors. Electrochimica Acta, 55, 7516-23.

[9] Devillers, N., Jemei, S., Péra, M-C., Bienaimé, D., Gustin, F. (2014). Review of characterization methods for supercapacitor modelling. J. Power Sources, 246, 596-608.

[10] Kuparowitz, T., Sedláková, V., Szewczyk, A., Hasse, L., Smulko, J. (2014). Charge Redistribution and Restoring voltage of Supercapacitors. Electroscope, 2014, 1-7.

[11] Ike, I.S., Sigalas, I., Iyuke, S., Ozoemena, K.I. (2015) An overview of mathematical modeling of electrochemical supercapacitors/ultracapacitors. J. Power Sources, 273, 264-77. 